Preprint typeset in JINST style - HYPER VERSION

\title{
Sub-millimeter nuclear medical imaging with high sensitivity in positron emission tomography using $\beta^{+} \gamma$ coincidences
}

\author{
C. Lang ${ }^{a}$, D. Habs ${ }^{a, b}$, K. Parodi ${ }^{a}$ and P.G. Thirolf ${ }^{a *}$ \\ ${ }^{a}$ Fakultät für Physik (LSf. Medizinische Physik), Ludwig-Maximilians Universität München, Am \\ Coulombwall 1, D-85748 Garching, Germany \\ ${ }^{b}$ Max-Planck-Institut für Quantenoptik, Hans-Kopfermann-Str. 1, D-84748 Garching, Germany \\ E-mail: Christian.Lang@physik.uni-muenchen.de
}

\begin{abstract}
We present a nuclear medical imaging technique, employing triple- $\gamma$ trajectory intersections from $\beta^{+}-\gamma$ coincidences, able to reach sub-millimeter spatial resolution in 3 dimensions with a reduced requirement of reconstructed intersections per voxel compared to a conventional PET reconstruction analysis. This ' $\gamma$-PET' technique draws on specific $\beta^{+}$-decaying isotopes, simultaneously emitting an additional photon. Exploiting the triple coincidence between the positron annihilation and the third photon, it is possible to separate the reconstructed 'true' events from background. In order to characterize this technique, Monte-Carlo simulations and image reconstructions have been performed. The achievable spatial resolution has been found to reach ca. 0.4 $\mathrm{mm}$ (FWHM) in each direction for the visualization of a ${ }^{22} \mathrm{Na}$ point source. Only 40 intersections are sufficient for a reliable sub-millimeter image reconstruction of a point source embedded in a scattering volume of water inside a voxel volume of about $1 \mathrm{~mm}^{3}$ ('high-resolution mode'). Moreover, starting with an injected activity of $400 \mathrm{MBq}$ for ${ }^{76} \mathrm{Br}$, the same number of only about 40 reconstructed intersections are needed in case of a larger voxel volume of $2 \times 2 \times 3 \mathrm{~mm}^{3}$ ('highsensitivity mode'). Requiring such a low number of reconstructed events significantly reduces the required acquisition time for image reconstruction (in the above case to about $140 \mathrm{~s}$ ) and thus may open up the perspective for a quasi real-time imaging.
\end{abstract}

KEYWORDS: Image reconstruction in medical imaging, PET, PET/CT, Compton imaging.

\footnotetext{
${ }^{*}$ Corresponding author
} 


\section{Contents}

1. Introduction 1

2. Decay properties of PET isotopes 2

3. The $\gamma$-PET imaging technique

4. Simulation and reconstruction setup

5. Results 7

5.1 Spatial source reconstruction resolution 7

5.2 Efficiency considerations 8

5.3 Sensitivity considerations 11

6. Conclusion and Outlook 12

\section{Introduction}

Functional medical imaging via positron emission tomography (PET) experienced an enormous growth in the last decade due to the large variety of different tracers and due to the significant improvement of the imaging performance [1]]. These tracers are chemical compounds, carrying a positron emitting isotope to, e.g., a tumor site. Annihilation of the positron into two back-to-back $511 \mathrm{keV}$ photons allows to restrict the source origin in 2 dimensions onto a line of response (LOR). Superimposing the LORs of different decay events locates the source distribution of the emitter, i.e. the concentration of the tracer molecules in a patient in 3D. Modern whole-body systems are combined PET/CT scanners, where CT provides the anatomical information for a reference frame and also data for the attenuation and scattering correction, while PET provides the molecular information. Such scanners are able to reach a spatial resolution of about 5-6 mm [2, 3]. The performance of new generation PET/CT with point-spread-function (PSF) correction can go down to $2.0 \mathrm{~mm}$ for the PET modality [4]. An improvement of conventional PET imaging is the Timeof-Flight (TOF) PET technique, where the flight time difference of the two annihilation photons registered in the responding pair of detectors is taken into account for achieving an improved spatial information. Annihilation events can be restricted to a certain area of the LOR, thus achieving an improved image quality by an improved signal-to-noise ratio. Also the patient dose and the examination time can be reduced. Moreover, especially biomedical research, using animal models of metabolism and disease mechanisms, would profit from a high-resolution small-animal PET device, where presently a resolution of $1.1 \mathrm{~mm}$ (FWHM) in the center of the field-of-view (FOV) has been reported, which degrades to $2.2 \mathrm{~mm}$ (FWHM) at a position with $2 \mathrm{~cm}$ radial offset [5, 7]. 
Even sub-millimeter spatial resolution may be reachable, as, e.g., first tests with continuous LYSO crystals coupled to a silicon photomultiplier matrix have shown [6]. Also the concept of a Compton camera has been investigated for its applicability with the PET technique, however, so far only at the laboratory development level [8]. In this case, position and energy information of the Compton scattering kinematics are measured in a scatter and an absorber detector, together allowing for a reconstruction of the direction to the source position on the surface of the 'Compton cone'. In general, the spatial resolution of a PET device is limited by several effects. Limiting factors are the spatial, temporal or energy resolution of the detectors, random coincidences or the resolving power of the source image reconstruction algorithm. Moreover, there are also inherent physical limits to the achievable image resolution, like Compton scattering of the $511 \mathrm{keV}$ photons within the patient or biological sample or the diffusion range of the positron before its annihilation, presenting the dominant limitation in small-animal PET imaging. Another inherently limiting factor is the acollinearity of the positron annihilation, i.e. the angular deviation from $180^{\circ}$ between the two annihilation photons, originating from the momentum distribution of the annihilating electronpositron pair, after thermalization of the positron (within a few ps) and positronium formation. Because of the thermalization of the positron prior to its annihilation, the acollinearity is mainly caused by the significantly higher momentum of the bound orbital electrons. In a recent study [9] the acollinearity describes the angular deviation from $180^{\circ}$ by two components: a broad main component originating from orbital electrons with $\Delta \theta=0.633(8)^{\circ}$ and a narrower component with $\left.\Delta \theta=0.27(10)^{\circ}\right)$ resulting from positronium annihilation. This corresponds to a spatial deviation of $2 \mathrm{~mm}$ in an average PET-ring radius of $40 \mathrm{~cm}$ and thus represents the dominant limiting factor for the spatial resolution of whole-body PET systems [10]. Another effect arising from the momentum distribution of the annihilating electron-positron pair is the Doppler broadening of the annihilation spectrum. The actual broadening depends on the annihilation medium and can be used as a measure for the longitudinal component of the momentum distribution [29, 30]. Besides the achievable spatial source reconstruction resolution, also the applied radioactive dose to the patient or sample, as well as the corresponding examination time, has to be taken into account when discussing medical imaging techniques. The applied radioactivity typically used in human PET studies is tracer specific and ranges from about 185 to $1850 \mathrm{MBq}$ [11], while in small-animal PET even higher activities are applied. A typical human PET examination using the radioisotope ${ }^{18} \mathrm{~F}$ takes $10-45$ minutes depending on scanner, tumor and image reconstruction method. While on the one hand such long examination times limit the number of patients' access to PET devices, they are prohibitive for real-time metabolism studies, due to, e.g., organ movements. Therefore, this study was motivated not only by aiming at an improved spatial resolution for PET examinations, but, perhaps even more attractive, by targeting a higher sensitivity via a lower reconstruction statistics required per voxel of the examination volume. Moreover, the technique described in the following also bears the potential to be applicable for ion beam range monitoring in hadron therapy [12, 13], exploiting the online generation of $\beta^{+}$emitting isotopes.

\section{Decay properties of PET isotopes}

Table 1 compares the decay properties of various presently used or potential future PET radioisotopes. The two isotopes ${ }^{10} \mathrm{C}$ and ${ }^{14} \mathrm{O}$ have been included here, despite of their short halflives of 
$19.3 \mathrm{~s}$ and $70.6 \mathrm{~s}$, respectively, since both can be produced during hadron therapeutic irradiations using a carbon or proton beam [14, 15]. Thus they qualify as candidates for online ion-beam range monitoring during therapy treatment. Moreover, proposals have been presented to directly use positron emitter beams, such as ${ }^{11} \mathrm{C}[16,17,18],{ }^{10} \mathrm{C}[19]$ or ${ }^{15} \mathrm{O}[20]$ as therapeutic beams, allowing for fast online ion-beam range verification. In Tab. 1 in particular the positron diffusion range in water has been simulated with Geant4 (last column) and benchmarked against measured values (column 6), showing good agreement. We used Geant4 (9.4) with the QGSP-BIC-HP physics list

\begin{tabular}{|c|c|c|c|c|c|c|}
\hline Isotope & $\begin{array}{l}\text { decay } \\
\text { mode }\end{array}$ & $\begin{array}{c}E_{e^{+}}^{\max } \\
{[\mathrm{MeV}]} \\
21]\end{array}$ & $\begin{array}{l}{[\%]} \\
{[21]}\end{array}$ & $\begin{array}{c}{[\mathrm{MeV}]} \\
{[21]}\end{array}$ & $\begin{array}{c}\text { mean range } \\
\text { in water } \\
\text { [mm] } \\
\text { (experiment, } \\
\text { [22]) }\end{array}$ & $\begin{array}{c}\text { mean range } \\
\text { in water } \\
{[\mathrm{mm}]} \\
\text { (simulation, } \\
\text { this work) }\end{array}$ \\
\hline${ }^{22} \mathrm{Na}$ & $\beta^{+}+\gamma$ & 0.54 & 100 & 1.27 & & $1.5 \pm 0.1$ \\
\hline${ }^{18} \mathrm{~F}$ & $\beta^{+}$ & 0.63 & 96.7 & & 1.4 & $1.4 \pm 0.1$ \\
\hline${ }^{94} \mathrm{Tc}$ & $\beta^{+}+\gamma$ & $0.81 / 1.83$ & $10.5 / 70.8$ & 0.87 & & $1.4 \pm 0.1$ \\
\hline${ }^{11} \mathrm{C}$ & $\beta^{+}$ & 0.96 & 99.8 & & 1.7 & $1.8 \pm 0.1$ \\
\hline${ }^{13} \mathrm{~N}$ & $\beta^{+}$ & 1.20 & 100 & & 2.0 & $1.9 \pm 0.1$ \\
\hline${ }^{44} \mathrm{Sc}$ & $\beta^{+}+\gamma$ & 1.47 & 94.3 & 1.16 & & $2.1 \pm 0.1$ \\
\hline${ }^{15} \mathrm{O}$ & $\beta^{+}$ & 1.73 & 99.9 & & 2.7 & $2.6 \pm 0.1$ \\
\hline${ }^{14} \mathrm{O}$ & $\beta^{+}+\gamma$ & 1.81 & 99.2 & 2.31 & & $2.6 \pm 0.1$ \\
\hline${ }^{68} \mathrm{Ga}$ & $\beta^{+}+\gamma$ & 1.90 & 88.0 & 1.08 & & $2.7 \pm 0.1$ \\
\hline${ }^{124} \mathrm{I}$ & $\beta^{+}+\gamma$ & $1.53 / 2.14$ & $11.7 / 10.8$ & 0.60 & & $2.9 \pm 0.1$ \\
\hline${ }^{10} \mathrm{C}$ & $\beta^{+}+\gamma$ & 2.93 & 98.5 & 0.72 & & $2.6 \pm 0.1$ \\
\hline${ }^{152} \mathrm{~Tb}$ & $\beta^{+}+\gamma$ & $2.62 / 2.97$ & $5.5 / 6.2$ & 0.34 & & $3.6 \pm 0.1$ \\
\hline${ }^{86} \mathrm{Y}$ & $\beta^{+}+\gamma$ & $\begin{array}{l}1.22 / 1.55 / \\
1.99 / 3.14\end{array}$ & $\begin{array}{c}11.9 / 5.6 \\
3.6 / 2.0\end{array}$ & 1.08 & & $2.3 \pm 0.1$ \\
\hline${ }^{76} \mathrm{Br}$ & $\beta^{+}+\gamma$ & $\begin{array}{l}0.87 / 0.99 \\
3.38 / 3.94\end{array}$ & $\begin{array}{c}6.3 / 5.2 \\
25.8 / 6.0\end{array}$ & 0.56 & & $4.1 \pm 0.1$ \\
\hline${ }^{82} \mathrm{Rb}$ & $\beta^{+}+\gamma$ & 4.39 & 100 & 0.78 & & $4.9 \pm 0.1$ \\
\hline
\end{tabular}

Table 1. Decay properties of presently used or potential future PET isotopes. The positron diffusion range has been simulated with Geant4 (last column) and compared to experimentally measured values, where available.

for hadronic interactions and the Livermore physics list for electromagnetic interactions [23].

In case of the positron range simulations, we placed the detector as close as possible to a water sphere of $6 \mathrm{~cm}$ diameter, thus ensuring to be in the regime where diffusion of the positron prior to its annihilation is the dominant factor for the position resolution, like in small-animal PET devices. ${ }^{22} \mathrm{Na}$ is the only non-medical radioisotope listed in Tab. 1 due to its use in our laboratory as test source for the later-on discussed $\gamma$-PET technique. 


\section{The $\gamma$-PET imaging technique}

The novel ' $\gamma$-PET' imaging technique presented here draws on specific $\mathrm{e}^{+}$sources, simultaneously emitting an additional photon with the $\beta^{+}$decay. Exploiting the triple coincidence between the positron annihilation and the additionally emitted photon, it is possible to efficiently separate the reconstructed 'true' events from background [8, 24]. Therefore, the image reconstruction sensitivity can be significantly increased by an improved signal-to-noise ratio, achieved via exploiting the spatial and temporal coincidence with the additionally emitted photon.

Radioisotopes like ${ }^{94(m)} \mathrm{Tc},{ }^{67} \mathrm{Br},{ }^{124} \mathrm{I},{ }^{86} \mathrm{Y},{ }^{152} \mathrm{~Tb},{ }^{52} \mathrm{Mn},{ }^{82} \mathrm{Rb}$ and ${ }^{44} \mathrm{Sc}$ are suitable candidates for the $\gamma$-PET technique (see Tab. 1 for details). Especially ${ }^{44} \mathrm{Sc}$ is of interest, which $\beta^{+}$-decays into the stable ${ }^{44} \mathrm{Ca}$, emitting an $1157 \mathrm{keV}$ photon. It has already been tested clinically [26]. With a short half-life of $3.9 \mathrm{~h}$ it has to be produced from a ${ }^{44} \mathrm{Ti}$ generator $\left(\mathrm{t}_{1 / 2}=60.4 \mathrm{a}\right)$ [25], which presently cannot be performed in clinically relevant quantities. However, this may change with the soon expected availability of highly brilliant $\gamma$ beams [24]. Due to the kinematics of the Compton scattering process and subsequent photon absorption, a Compton camera allows for reconstructing the origin of a primary photon on the surface of the 'Compton cone'. Superimposing different cones from different events reduces the reconstructed source distribution in 3 dimensions to the few-millimeter range [27]. The $\gamma$-PET technique is different, as it will intersect the Compton cone with the line of response (LOR) from the same $\beta^{+}$annihilation $\gamma$ coincidence event, thus allowing to reconstruct the source distribution in 3 dimensions from individual events. The principle of the $\gamma$-PET technique can be seen in the left panel of Fig. 1. The emitted $\gamma$ ray will first be Comptonscattered in a position-sensitive double-sided silicon strip detector (DSSSD). Subsequently, the $\gamma$ ray will be absorbed in a position-sensitive $\mathrm{LaBr}_{3}$ scintillator, again measuring position and energy of this final interaction. The $\mathrm{e}^{+}$annihilation into two (almost) back-to-back $511 \mathrm{keV}$ photons defines the LOR. The intersection of the Compton cone with the LOR restricts the source origin in 3 dimensions within one $\beta^{+} \gamma$ coincidence event, as shown in Fig. 1 $1 \mathrm{~b}$ ). Figure 1 1 a) displays the schematical geometry of a $\gamma$-PET setup as used in our simulations, consisting of four Compton cameras, placed around a $\beta^{+}$source isotope emitting a positron and a prompt $\gamma$ ray. The energy loss $\Delta E_{\gamma, 1}$, the residual energy $\mathrm{E}_{\gamma, 2}$ and the interaction positions of the Compton scattering process of the prompt $\gamma$ are measured in a double-sided silicon strip detector (DSSSD) and an absorbing scintillator $\left(\mathrm{LaBr}_{3}\right)$, respectively. The initial photon energy $\mathrm{E}_{\gamma, 1}$ can be calculated from summing $\Delta E_{\gamma, 1}$ and $\mathrm{E}_{\gamma, 2}$. The opening angle $\theta$ of the Compton-scattering cone can be derived according to

$$
\cos \theta=1-m_{e} c^{2} \frac{E_{\gamma, 2}}{E_{\gamma, 1}\left(E_{\gamma, 1}-E_{\gamma, 2}\right)} .
$$

This formula (in contrast to the MC simulations) is not taking into account the effect of Doppler broadening, arising from the non-zero momentum of the bound electron. The Doppler broadening contributes to the physical limits of the achievable angular resolution of a Compton camera [28]. In case of an unknown initial photon energy $\mathrm{E}_{\gamma, 1}$, which could occur if the $\beta^{+} \gamma$-emitters were produced during hadron therapy with, e.g., a ${ }^{12} \mathrm{C}$ beam (potentially generating different $\beta^{+} \gamma$-emitters like ${ }^{10} \mathrm{C}$ or ${ }^{14} \mathrm{O}$ ), proper event and image reconstruction would require a full absorption of $\mathrm{E}_{\gamma, 2}$ in the scintillator. The intersection (Fig. 1]b) of the Compton cone and the LOR strongly suppresses background and restricts the reconstructed events to those belonging to the same $\beta^{+} \gamma$ coincidence 
a)

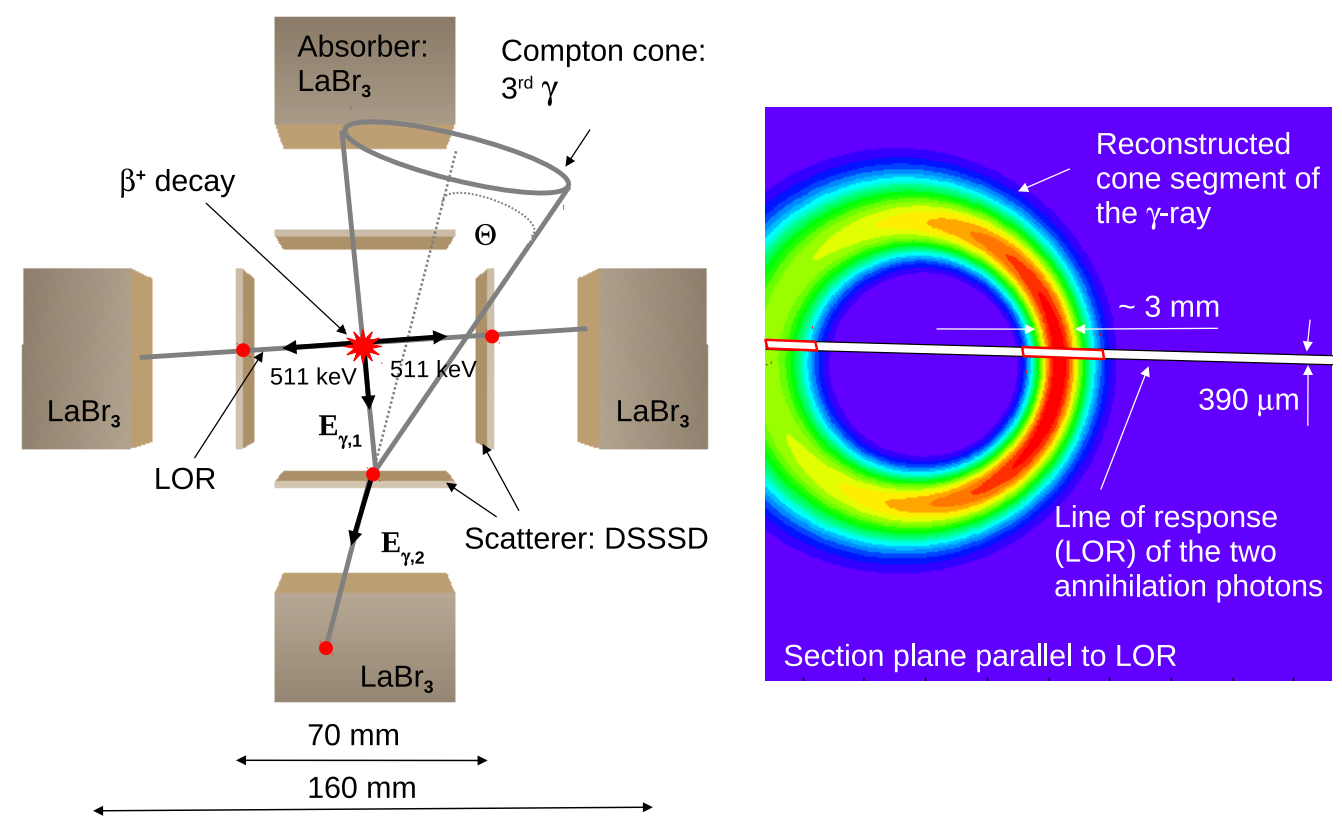

Figure 1. Principle of the $\gamma$-PET technique (for details see text).

event, originating from a volume defined by the displacement between the positions of the $\beta^{+}$ decay and the positron annihilation, depending on the time resolution of the detector system. In contrast to the restriction of the photon emission volume, the acollinearity effect cannot be removed by the $\gamma$-PET technique. Also Compton scattering of the $511 \mathrm{keV}$ photons within the patient limits the performance. A further improvement of the $\gamma$-PET technique (so far not implemented in our reconstruction code) would take attenuation and scatter corrections of the annihilation photons, defining the LOR, into account [31, 32] (while scattering effects have been correctly simulated).

\section{Simulation and reconstruction setup}

In order to characterize the spatial resolution of a PET scanner, a Derenzo phantom is commonly used [33]. We simulated a quasi-Derenzo phantom (see Fig. 2] for a sketch of the source geometry), consisting of twelve ${ }^{22} \mathrm{Na}$ point sources with $100 \mathrm{kBq}$ activity each. Point sources were chosen for this exploratory study, while in a later stage it is foreseen to extend this to the realistic scenario of an extended source. Four equilateral triangles are arranged in four sections, containing 3 point sources each, with a separation of $0.2 \mathrm{~mm}, 0.4 \mathrm{~mm}, 0.6 \mathrm{~mm}$ and $0.8 \mathrm{~mm}$, respectively. This source arrangement was placed inside a water sphere of $6 \mathrm{~cm}$ diameter to imitate a medical or biological sample and to take into account photon scattering effects in the sample during the source reconstruction. Each of the four Compton camera modules consists of a $\mathrm{LaBr}_{3}$ scintillator crystal $\left(50 \times 50 \times 30 \mathrm{~mm}^{3}\right)$, read out by a 2D-segmented photomultiplier with 64 pixels $\left(6 \times 6 \mathrm{~mm}^{2}\right.$ each). 


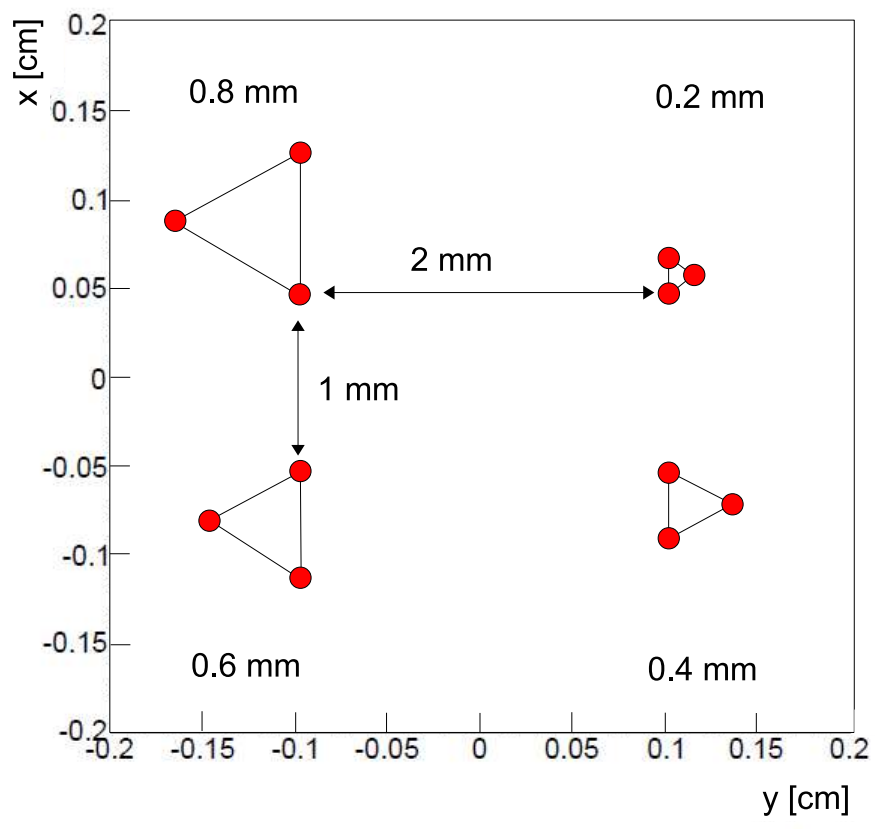

Figure 2. Sketch of the simulated source geometry, representing a quasi-Derenzo phantom [33], consisting of twelve ${ }^{22} \mathrm{Na}$ point sources with $100 \mathrm{kBq}$ activity each.

An energy threshold of $5 \mathrm{keV}$ (i.e. exceeding the electronic noise level) and an energy resolution varying from $\Delta \mathrm{E} / \mathrm{E}=4.7 \%$ at $500 \mathrm{keV}$ to $3.5 \%$ at $1 \mathrm{MeV}$ were used. Furthermore, a double-sided silicon strip detector with 128 strips on each side, an active area of $50 \times 50 \mathrm{~mm}^{2}$ and a thickness of $2 \mathrm{~mm}$ was used as scatterer. The pitch size of $390 \mu \mathrm{m}$ correspondingly leads to a width of the LOR of $390 \mu \mathrm{m}$ (FWHM). An energy resolution of $10 \mathrm{keV}$ (FWHM) and a detection threshold of $10 \mathrm{keV}$ in the Monte Carlo simulation (due to the assumed electronic noise level of the DSSSD) was chosen. For the detector system a time resolution of $1 \mathrm{~ns}$ was (conservatively) assumed. The rise time of signals from the $\mathrm{LaBr}_{3}$ scintillator is about $8 \mathrm{~ns}$, resulting in a time resolution of $560 \mathrm{ps}$ for our $50 \times 50 \times 30 \mathrm{~mm}^{3}$ crystal. With a typical signal rise time of around $2 \mathrm{~ns}$ for the silicon strip detector, the time resolution of the combined detector system can be expected to be even faster than $1 \mathrm{~ns}$. In order to test the feasibility of the $\gamma$-PET technique, Monte-Carlo simulations and image reconstruction have been performed using the 'Medium Energy Gamma-Ray Astronomy' library MEGAlib [28]. MEGAlib is a software framework designed to simulate and analyze data from Compton cameras. The library consists of a Monte-Carlo simulation package, which utilizes the ROOT and Geant4 (9.4) software library, an event reconstruction and an image reconstruction section based on a list-mode maximum likelihood expectation maximization algorithm (LM-MLEM). This algorithm is an iterative method to reconstruct the most probable source distribution. For the requirements of the $\gamma$-PET technique, we modified MEGAlib to realize an event reconstruction from the intersection between the Compton cone and the LOR. Subsequently, after successful event reconstruction, this information serves as starting point for an iterative image reconstruction of the $\gamma$-source positions. 


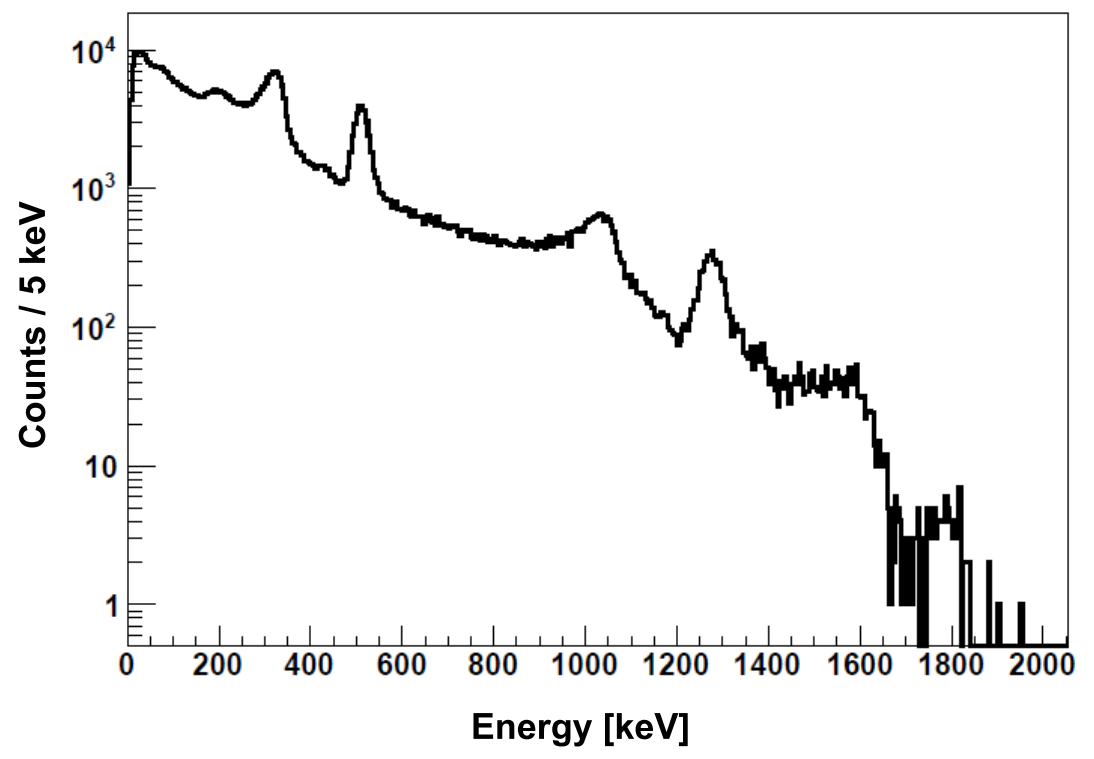

Figure 3. $\gamma$-ray energy spectrum emitted from the twelve ${ }^{22} \mathrm{Na}$ point sources, as detected in one of the 4 Compton camera modules placed outside a water sphere of $6 \mathrm{~cm}$ diameter. The point sources were arranged in the geometry of the Geant4 Monte-Carlo simulation as indicated in Fig. 2.

\section{Results}

In the following we address the potential of the proposed $\gamma$-PET imaging technique by separately discussing the main issues of spatial resolution, detection efficiency and reconstruction sensitivity for different arrangements of the detection system and imaged isotope.

\subsection{Spatial source reconstruction resolution}

The $\gamma$-ray energy spectrum, as emitted from the twelve ${ }^{22} \mathrm{Na}$ point sources, and detected by one Compton camera module (including the detector resolution), placed outside a water sphere of 6 $\mathrm{cm}$ diameter, is shown in Fig. 3. The spectrum was obtained from a Monte-Carlo simulation using Geant4 (9.4).

The decrease of of the $\gamma$-ray yield with energy is due to incompletely absorbed $\gamma$ rays. Clearly visible are the $511 \mathrm{keV}$ positron annihilation line, as well as the $1275 \mathrm{keV}$ line from the $\gamma$ ray of the $\beta^{+}$decay of ${ }^{22} \mathrm{Na}$. The strong contribution at $340 \mathrm{keV}$ comes from electrons due to Compton backscattering of $511 \mathrm{keV}$ while the peak at $1062 \mathrm{keV}$ originates from Compton-backscattered $1275 \mathrm{keV}$ photons. The trigger condition in these simulations required three hits in three of the DSSSD modules and one hit in one of the the $\mathrm{LaBr}_{3}$ absorbers. The line at $1786 \mathrm{keV}$ is due to pileup between the $1275 \mathrm{keV}$ transition and one of the $511 \mathrm{keV}$ annihilation photons. Based on the geometrical arrangement of ${ }^{22} \mathrm{Na}$ sources (Fig. 2) and detector modules (Fig. 17a), the underlying data of the detected $\gamma$-ray energy spectrum (Fig. 3) are first used for a kinematical event reconstruction. The event reconstruction identifies Compton events in an energy window of $1275 \pm 50$ 


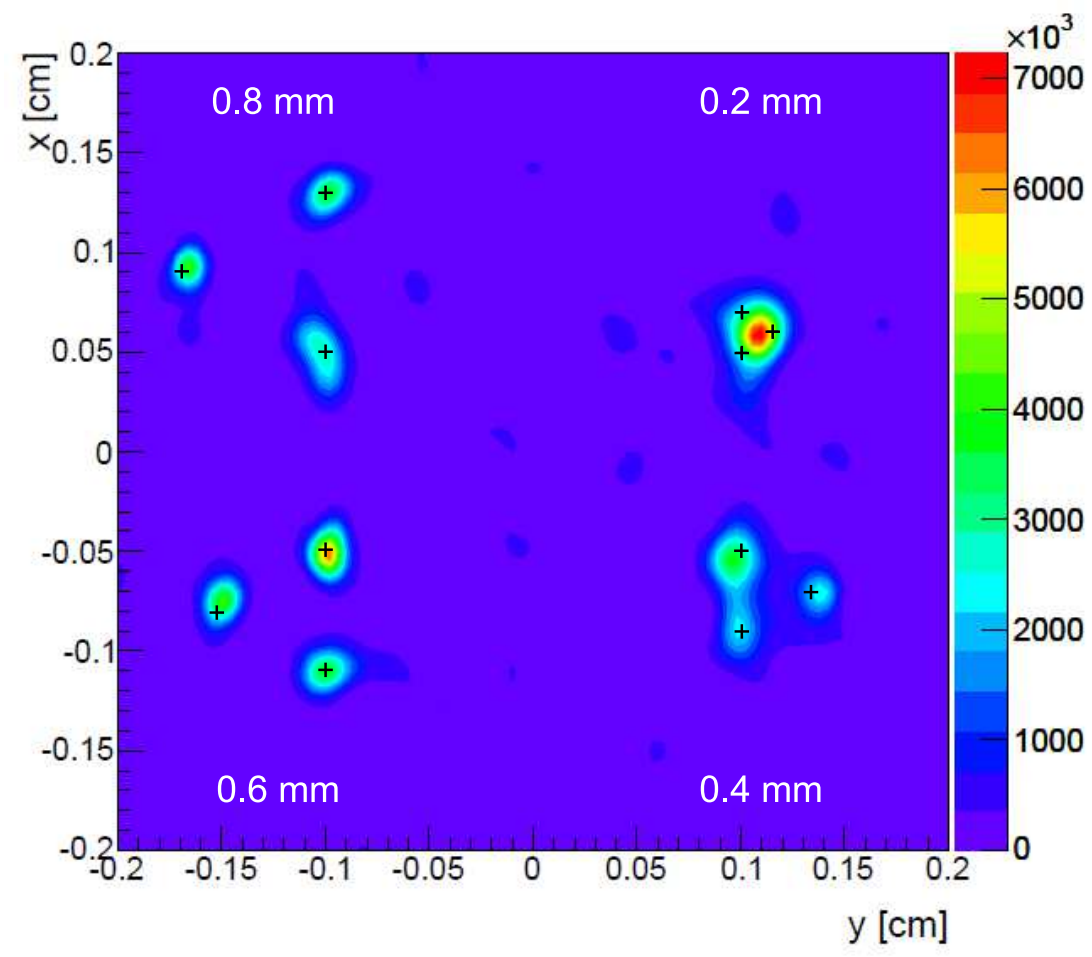

Figure 4. Image of the reconstructed $\gamma$-source geometry of the quasi-Derenzo phantom introduced in Fig. 2, using the $\gamma$-PET technique after 100 iterations (depending on start parameters) using a maximum-likelihood algorithm. The black crosses indicate the original source positions.

$\mathrm{keV}$, corresponding to the $\gamma$-ray energy from the ${ }^{22} \mathrm{Na}$ decay, also identifying simultaneous hits above the detection threshold in the DSSSD for reconstruction of the LOR. In Fig. 4 , the resulting image of the reconstructed $\gamma$-source geometry is shown, as obtained from exploiting the $\gamma$-PET technique. It was possible to clearly resolve the two largest triangles with spacings of $0.8 \mathrm{~mm}$ and $0.6 \mathrm{~mm}$, respectively. The triangle with $0.4 \mathrm{~mm}$ spacing still could be resolved sufficiently well, while the $0.2 \mathrm{~mm}$ spaced triangle could not be resolved at all. The black crosses indicate the original source positions in the simulation. Due to the $\gamma$-PET technique, the imaging sensitivity for positron annihilation significantly displaced from the initial decay spot via thermalization and diffusion is strongly suppressed, and only positron annihilation photons emitted in spatial and temporal coincidence with the third (prompt) $\gamma$ are included for image reconstruction. While the acollinearity of annihilation photons in our close detector geometry (distance $50 \mathrm{~mm}$ to the source) contributes only about $0.3 \mathrm{~mm}$ to the position uncertainty, Fig. 5 shows the correlation between the spatial resolution (as estimated via the above described quasi-Derenzo phantom) and the $\beta$ end-point energy $E_{e^{+}}^{\max }$ for the three isotopes ${ }^{22} \mathrm{Na},{ }^{44} \mathrm{Sc}$ and ${ }^{10} \mathrm{C}$. Isotopes with $E_{e^{+}}^{\max }<4 \mathrm{MeV}$ are promising candidates for sub-millimeter imaging in our geometrical detector arrangement.

\subsection{Efficiency considerations}

After having shown that the $\gamma$-PET technique allows for sub-millimeter spatial resolution in the position reconstruction of the underlying radio-tracer independent of its $\beta^{+}$energy, we address a 


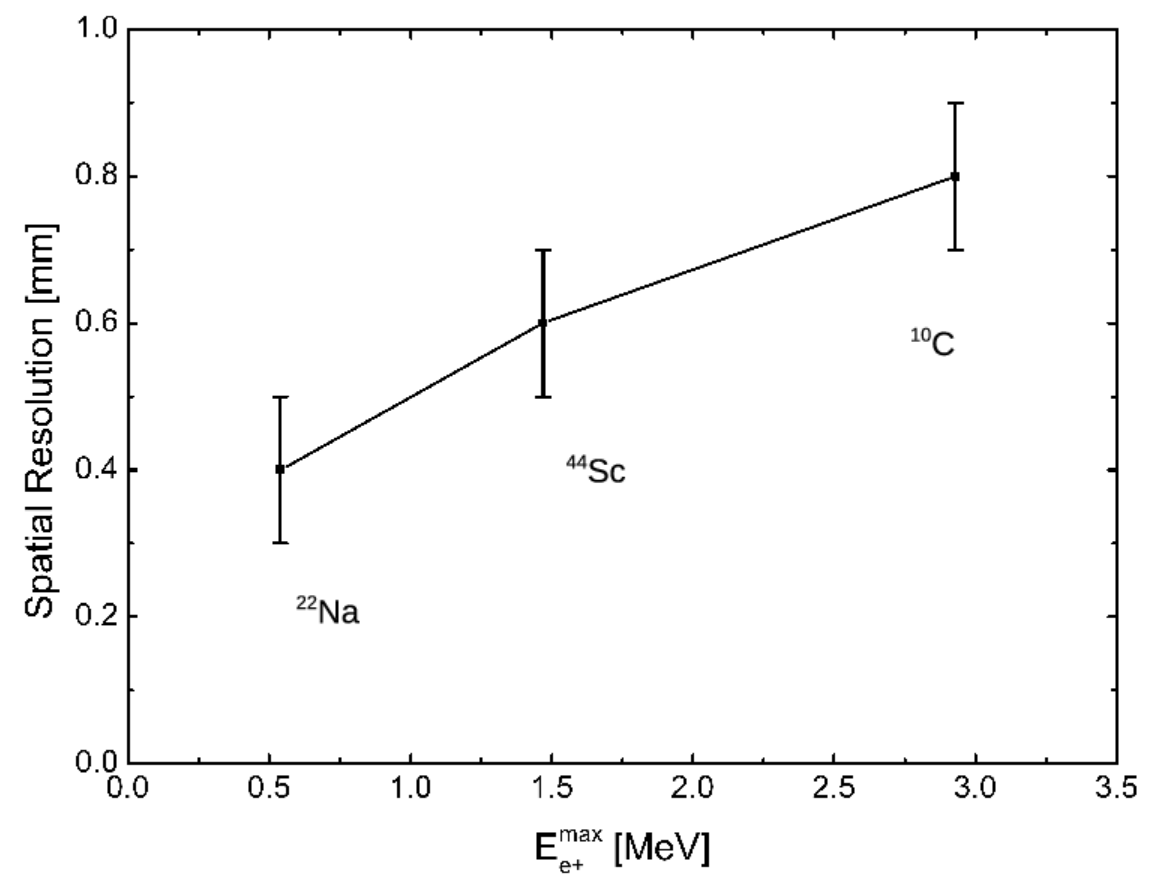

Figure 5. Correlation between the spatial resolution (as estimated from an image reconstruction using the quasi-Derenzo phantom described before) and the $\beta$ end-point energy $E_{e^{+}}^{\max }$ for the isotopes ${ }^{22} \mathrm{Na},{ }^{44} \mathrm{Sc}$ and ${ }^{10} \mathrm{C}$.

further major advantage of this method, which is the acquisition time reduction achievable with the highly sensitive triple- $\gamma$ coincidence measurement.

During the analysis of the $511 \mathrm{keV}$ annihilation photons, no energy conditions have been applied. The simulations showed that there is no necessity for a stricter event definition than requiring an energy deposit (within a coincidence time window of $1 \mathrm{~ns}$ ) above a threshold of $10 \mathrm{keV}$ in three DSSSD detectors (two of them diametral) and a (completely absorbed) $1275 \mathrm{keV}$ (prompt gamma from ${ }^{22} \mathrm{Na}$ decay) signal from the summed signal of the third scatterer and its scintillator to generate the LOR and still reach sub-millimeter spatial resolution. This can mainly be attributed to the capability of the event reconstruction algorithm to provide a reliable reconstruction even on the basis of an incomplete photon energy absorption. Additional energy conditions would discard this latter class of events (containing 5.8 $10^{-2}\left(3.4 \cdot 10^{-3}\right)$ of all events for one (two) incompletely absorbed $511 \mathrm{keV}$ photon(s)) and unnecessarily lead to a drastic reduction of the reconstruction efficiency (see below). Here, 'reconstruction efficiency' denotes the ratio of the number of identified intersections between the LOR of the positron annihilation photons and the Compton cone derived from the detection of the third prompt photon and the number of initial decay events. It comprises contributions from the photon detection efficiency as well as from the image reconstruction sensitivity. Moreover, the narrow timing coincidence of $1 \mathrm{~ns}$ significantly helps to remove random background. One individual Compton camera module simulated here provides an event reconstruction efficiency of $3.3 \cdot 10^{-5}$. Thus the geometry studied with 4 camera modules exhibits an overall reconstruction efficiency for the Compton cone of $1.3 \cdot 10^{-4}$. Moreover, a 5 (8) times thicker scatterer (or a stack of 5 (8) scatter detectors with a summed thickness of 10 (16) $\mathrm{mm}$ ) per 
Compton camera module would increase the reconstruction efficiency of the Compton cones by an additional factor of $4.4(5.8)$ to a value of $5.7(7.5) \cdot 10^{-4}$. Similar scatter detector thicknesses have been favoured in [34]. In the case of thicker scatterer, where no depth information of the scattering point is measured, the spatial resolution increases from $0.4 \mathrm{~mm}$ (for $2 \mathrm{~mm}$ scatterer) to $0.6 \mathrm{~mm}$ (for $16 \mathrm{~mm}$ scatterer). This efficiency could be further increased by replacing our prototype geometry with a pyramidal arrangement of a scatter detector and a larger absorber, covering the opening angle of the cone seen from the photon source at the top of the pyramid. For our detector geometry, this would require an absorber with an area of $114 \times 114 \mathrm{~mm}^{2}$, about 5 times larger than the one used in our study.

Finally, when extending the Compton camera to a $\gamma$-PET device, the temporal and spatial coincidence with the annihilation LOR has to be considered. The simulated triple-coincidence detection efficiency for the $\gamma$-PET technique (assuming the detector setup of Fig. 17a)) amounts to $7.0 \cdot 10^{-8}$ reconstructed intersections per ${ }^{22} \mathrm{Na}$ decay between the LOR of the annihilation photons and the Compton cone of the third photon. This reduction of the above given Compton camera efficiency is on the one hand due to the solid angle acceptance of the scatter detectors entering the LOR reconstruction, in our scenario resulting in a geometrical coincidence probability of 0.026 . Moreover, a loss of those events has to be considered, where due to the diffusion of the positron before its annihilation no intersection between its reconstructed LOR and the Compton cone of the third photon, i.e. a spatial and temporal coincidence, could be found. This fraction amounts to ca. $91.8 \%$, in total resulting in the above given overall $\gamma$-PET reconstruction efficiency of $7.0 \cdot 10^{-8}$ for ${ }^{22} \mathrm{Na}$. This value would be further reduced to a prohibitively low value of $1.1 \cdot 10^{-10}$, if in addition to the condition set to the energy deposition of $1275 \mathrm{keV}$ in the scatterer and absorber from the third photon also conditions on the energy of the two diametral $511 \mathrm{keV}$ annihilation quanta (besides their temporal coincidence within $1 \mathrm{~ns}$ ) would have been required. In a pyramidal arrangement of a scatter detector and a larger absorber covering the full opening angle of the scatterer as seen from the emission point, such an additional energy condition would not reduce the efficiency, but would reduce random coincidences. For an optimized detector system consisting of 4 Compton camera modules, each with $8 \times 2 \mathrm{~mm}$ thick scatter detectors and geometrically matched sizes of the absorber crystals, a triple reconstruction efficiency of $9.7 \cdot 10^{-5}$ (for ${ }^{22} \mathrm{Na}$ ) can be expected. The efficiencies of other isotopes are different, due to the individual $\gamma$ energies $E_{\gamma}$, the different positron endpoint energies $E_{e^{+}}^{\max }$ and the branching ratio of $\gamma / \beta^{+}$.

The simulated reconstruction efficiencies for various $\beta^{+} \gamma$ emitters are listed in Tab. 2 .

\begin{tabular}{l|cccccccc} 
Isotope & ${ }^{22} \mathrm{Na}$ & ${ }^{44} \mathrm{Sc}$ & ${ }^{14} \mathrm{O}$ & ${ }^{68} \mathrm{Ga}$ & ${ }^{124} \mathrm{I}$ & ${ }^{10} \mathrm{C}$ & ${ }^{76} \mathrm{Br}$ & ${ }^{82} \mathrm{Rb}$ \\
\hline$\varepsilon_{\text {rec }, 1}\left[10^{-8}\right]$ & 7.0 & 6.0 & 1.6 & 0.093 & 20 & 6.8 & 47 & 2.6 \\
\hline$\varepsilon_{\text {rec }, 2}\left[10^{-5}\right]$ & 9.7 & 8.0 & 5.1 & 0.13 & 22 & 2.8 & 89 & 3.3 \\
\hline
\end{tabular}

Table 2. Simulated reconstruction efficiencies for various $\beta^{+}-\gamma$ emitters. In case of ${ }^{124} \mathrm{I}$ and ${ }^{76} \mathrm{Br}$, which emit several prompt $\gamma$ rays with considerable branching ratio, the individual efficiencies have been summed up ( ${ }^{124} \mathrm{I}: 0.60 \mathrm{MeV}, 0.72 \mathrm{MeV}, 1.5 \mathrm{MeV}$ and $1.7 \mathrm{MeV} .{ }^{76} \mathrm{Br}$ : $0.56 \mathrm{MeV}, 0.66 \mathrm{MeV}, 1.13 \mathrm{MeV}, 1.22 \mathrm{MeV}$ and $1.85 \mathrm{MeV}$ ). The upper line corresponds to the detector system shown in Fig. 1a), while the lower line represents the reconstruction efficiency for an optimized system with thicker scatter and larger absorber detectors (see text). 


\subsection{Sensitivity considerations}

A minimum of 40 reconstructed intersections between the LOR and the reconstructed emission direction of the third photon is sufficient for a reliable image reconstruction of a point source with a ratio of true-to-false reconstructed events allowing for a correct reconstruction of the (submillimeter) point-source position without fragmentation of the source image. Choosing a typical injected activity of $400 \mathrm{MBq}$ and taking into account our intersection reconstruction efficiency $\varepsilon_{\text {rec }, 2}$ for ${ }^{76} \mathrm{Br}$, 40 intersections can be identified after an examination time of about 140 seconds, which is sufficient for a reliable sub-millimeter image reconstruction of a point source contained within a voxel volume of about $1 \mathrm{~mm}^{3}$ ('high-resolution mode'). In a standard PET Iterative Reconstruction analysis (without exploiting time-of-flight information, i.e. ordered subset expectation maximization (OSEM) [35]), about 6000 true coincidences acquired with a Siemens Biograph mCT PET scanner [ [] are necessary to localize a ${ }^{22} \mathrm{Na}$ point source in the center of the scanner field-of-view using the smallest voxel volume of $2 \times 2 \times 3 \mathrm{~mm}^{3}$. In order to compare the performance of the $\gamma$ PET technique with conventional PET, we determined the imaging sensitivity of the method based on a comparable width of the LOR of ca. $2 \mathrm{~mm}$ (in contrast to the previously used value for ${ }^{22} \mathrm{Na}$ of $0.4 \mathrm{~mm}$ ). In such a case also about 40 reconstructed intersections per voxel (derived without iterative reconstruction procedure) lead to a reliable localization of the ${ }^{22} \mathrm{Na}$ point source. This sensitivity can be evaluated and compared to standard PET by quantifying the examination time required to localize a point source as described above. The minimum number of $\beta^{+}$-emitter decays per voxel $N_{\text {decay }}$, required for localizing a point source, relates to the imaging sensitivity, expressed by the minimum number of reconstructed intersections $N_{\text {inter }}$ and the corresponding reconstruction efficiency $\varepsilon_{\text {rec }}$ as well as to the activity concentration $C(i)$ of each voxel $i$, the examination time $\Delta t$ and the voxel volume $V_{\text {vox }}$ according to

$$
N_{\text {decay }}(i)=N_{\text {inter }}(i) / \varepsilon_{\text {rec }}=C(i) \cdot \Delta t \cdot V_{\text {vox }}(i) .
$$

For a given activity concentration and tumor size the required examination time for localizing a point source only depends on the imaging sensitivity $N_{\text {inter }}$ and the reconstruction efficiency $\varepsilon_{\text {rec }}$. While the $\gamma$-PET method described here features a clear advantage in terms of sensitivity expressed by $N_{\text {inter }}$ compared to standard PET, it falls behind when comparing the corresponding efficiencies $\varepsilon_{\text {rec }}$, where values of about 0.1 are reported for small-animal PET scanners [36], while similar values are found for whole-body scanners [37].

Tab. 3 lists in the last column the examination times $\tau^{\text {exam }}$ required for the localization of point sources of selected $\gamma$-PET radioisotopes, where information of tracer and associated equivalent dose coefficients $\left(\mathrm{h}_{T}\right)$ were available. $\tau^{\text {exam }}$ reflects the imaging sensitivity of the $\gamma$-PET method based on the minimum requirement of 40 reconstructed intersections and the isotope-specific reconstruction efficiencies $\varepsilon_{\mathrm{rec}, 2}$ (see Tab. 2). Here we assume a typical value for the activity concentration accumulated in a tumor of $25 \mathrm{kBq} / \mathrm{ml}$, corresponding to PET examination of a patient with a body weight of $80 \mathrm{~kg}$ with an injected dose of $400 \mathrm{MBq}$ and an average SUV (standard uptake value) of 5. Column 7 shows the corresponding effective dose values (calculated with $\mathrm{h}_{T}$ values derived for adult males). The rather wide spread of $\tau^{\text {exam }}$ values, reflecting the corresponding isotope-specific spread of the reconstruction efficiency $\varepsilon_{\text {rec }}$, exhibits no clear correlation with any of the isotopic properties like the $\beta^{+}$endpoint energy or the energy of the third prompt photon. Thus it may rather represent a correlated interplay between different factors that makes it 


\begin{tabular}{llcccccc} 
Isotope & $\begin{array}{l}\mathrm{t}_{1 / 2} \\
{[\mathrm{~min}]}\end{array}$ & $\begin{array}{c}\gamma / \beta^{+} \\
{[\%]}\end{array}$ & Tracer & $\begin{array}{c}\mathrm{h}_{T} \\
{[\mu \mathrm{Sv} / \mathrm{MBq}]}\end{array}$ & $\begin{array}{c}\text { Ref. } \\
\mathrm{E}^{\text {exam }} \\
{[\mathrm{mSv}]}\end{array}$ & $\begin{array}{c}\tau^{\text {exam }} \\
{[\mathrm{s}]}\end{array}$ \\
\hline${ }^{14} \mathrm{O}$ & 1.2 & 99 & Water & 0.88 & {$[38]$} & 0.33 & 2420 \\
${ }^{124} \mathrm{I}$ & 6013 & 90 & MIBG & 250 & {$[39]$} & 92 & 558 \\
${ }^{76} \mathrm{Br}$ & 16.2 & 100 & MAb-38S1 & 410 & {$[42]$} & 150 & 138 \\
${ }^{82} \mathrm{Rb}$ & 1.3 & 13 & Chloride & 1.28 & {$[40]$} & 0.46 & 3740 \\
${ }^{44} \mathrm{Sc}$ & 236 & & DOTATOC & n.a. & {$[25]$} & & 1100
\end{tabular}

Table 3. $\gamma$-PET examination time $\tau^{\text {exam }}$ for the localization of a point source of selected $\beta^{+}-\gamma$-decaying radioisotopes, assuming an injected activity of $400 \mathrm{MBq}$ and a tumor activity concentration of $25 \mathrm{kBq} / \mathrm{ml}$. $\tau^{\text {exam }}$ reflects the imaging sensitivity of the $\gamma$-PET method based on the minimum requirement of 40 reconstructed intersections and the isotope-specific reconstruction efficiencies $\varepsilon_{\mathrm{rec}, 2}$. Column 7 shows the corresponding effective dose $\mathrm{E}^{\text {exam }}$, based on the associated equivalent dose coefficients $\mathrm{h}_{T}$ (for adult males). The first columns list decay properties and suitable tracers of the respective isotopes.

difficult to predict the performance of a specific radioisotope when applying the $\gamma$-PET technique. In case of small-animal PET, where even higher effective doses are injected, real-time imaging of the metabolism or organ motion, or a study of biological washout diffusion processes in animal experiments with implanted radioisotopes [19], appears feasible with the $\gamma$-PET technique.

\section{Conclusion and Outlook}

Most medical radioisotopes typically give rise to a lower spatial resolution for PET imaging, compared to the most widely used ${ }^{18} \mathrm{~F}$, due to their higher $\beta^{+}$decay energies, resulting in a larger positron diffusion range. We investigated the ' $\gamma$-PET' imaging technique, taking advantage of detecting the additionally emitted $\gamma$ ray in coincidence with the $\beta^{+}$annihilation photons. The triplecoincidence measurement allows to reduce the image-blurring effect of the diffusion range of the positron prior to its annihilation, which increasingly gains importance when comparing radioisotopes with higher $\beta^{+}$endpoint-energies compared to the $634 \mathrm{keV}$ for ${ }^{18} \mathrm{~F}$. Since in analogy to PET, Compton scattering of the annihilation radiation within the patient limits the imaging performance, a further improvement of the $\gamma$-PET technique would have to take attenuation and scatter corrections of the annihilation photons, defining the LOR, into account [31], [32]. Simulations showed that it is possible to reach sub-millimeter spatial resolution in case of a small-animal imaging scenario, i.e. a small distance between the source and the detector, where the limiting influence of the acollinearity can be neglected. Even in case of high-energy positron emitting isotopes like ${ }^{76} \mathrm{Br}$ $\left(E_{e^{+}}^{\max }=3.38 \mathrm{MeV}\right)$ or ${ }^{10} \mathrm{C}\left(E_{e^{+}}^{\max }=2.93 \mathrm{MeV}\right)$, the image reconstruction will again result in submillimeter spatial resolution. Moreover, being left only with the limiting effect of the acollinearity for whole-body PET scanning, most of the radioisotopes listed in Tab. 1 still allow to reach submillimeter spatial resolution also for a clinical scenario.

One Compton camera module as described in Fig. 1, having a rather limited field-of-view, exhibits a Compton-cone reconstruction efficiency of $3 \cdot 3 \cdot 10^{-5}$. However, the $\gamma$-PET technique requires at least 3 camera modules. We simulated different detector arrangements for a $\gamma$-PET prototype consisting of 4 Compton camera modules, resulting for an optimized setup with thick scatter de- 
tectors and large absorbing crystals - matched to the solid angle covered by the scatterer - in an isotope-dependent triple-photon intersection reconstruction efficiency between $1.3 \cdot 10^{-6}\left({ }^{68} \mathrm{Ga}\right)$ and $8.9 \cdot 10^{-4}\left({ }^{76} \mathrm{Br}\right)$.

Particularly attractive is the highly sensitive image reconstruction capability provided by the $\gamma$ PET technique, found superior to a conventional PET scanners. Presently the full potential of this advantage cannot be exploited, due to the much reduced reconstruction efficiency compared to conventional full-body or small-animal PET scanners. Thus research and development efforts should be directed towards optimizing the efficiency achievable with $\gamma$-PET. Moreover, the present study was limited to the performance involving a point source in a scattering medium, while further work will also address the characterization of the method with respect to extended photon sources.

Finally, the Compton camera described here could also turn out to be beneficial in a therapeutic hadron beam irradiation, where $\beta^{+}(\gamma)$ emitters $\left({ }^{10} \mathrm{C},{ }^{14} \mathrm{O}\right)$ are generated via, e.g., the carbon beam. Especially the projectile (fragment) ${ }^{10} \mathrm{C}$ with its short half-life of $19.3 \mathrm{~s}$ and the quasi-simultaneous emission of a third photon from an excited state qualifies as an online marker isotope during hadron therapy. Its spatial distribution within the patient could be tomographically reconstructed, either from a (quasi-realtime) PET analysis (i.e. direct reconstruction using TOF-PET) or using the hybrid $\gamma$-PET technique to achieve an improved spatial resolution together with an enhanced sensitivity, i.e. reduced requirements to the signal strength. The presented Compton camera (eventually upgraded by a thicker scatterer), could provide a versatile setup to assist with targeting one of the crucial issues of hadron therapy, which is ion beam range verification, either by detecting prompt $\gamma$ radiation during the irradiation [43] or (delayed) short-lived $\beta^{+}$-decaying reaction products (PETor $\gamma$-PET operation) in between the irradiation cycles. Exploiting the perspectives of the $\gamma$-PET technique may thus allow to turn the present disadvantages of $\beta^{+} \gamma$-emitting PET isotopes into a benefit in resolution or sensitivity.

\section{Acknowledgments}

We are indebted to Christopher Kurz from the Heidelberg Radiooncology Department for his help and committment in deriving the statistics limits for conventional PET imaging. Also, fruitful discussions with Dr. Guido Böning from the LMU Nuclear Medicine Department are gratefully acknowledged. This work was supported by the DFG Cluster of Excellence MAP (Munich-Centre for Advanced photonics).

\section{References}

[1] P. Suetens, Fundamentals of Medical Imaging, Cambridge Univ. Press 2002.

[2] http://www.healthcare.philips.com/main/products/nuclearmedicine/products/geminitfbigbore/

[3] http://www.gehealthcare.com/eude/fun_img/products/pet_ct_imaging/products/discovery_st.html

[4] www.healthcare.siemens.com/siemens_hwem-hwem_ssxa_websites-context-root/wcm/i_dc /groups/public/@global/@imaging/@molecular/documents/download/mdaw/njcl/edisp /biograph_mct_brochure_3_13_f-00437492.pdf

[5] S.-J. Park et al., A Prototype of a Very High-Resolution Small Animal PET Scanner using Silicon Pad Detectors, Nucl. Instr. Meth. A 570, 543 (2007). 
[6] G. Llosa et al., Towards a Sub-Millimeter PET Prototype with Continuous LYSO Crystals and SiPM Matrices, Contrib. to IEEE NSS-MIC2012, Anaheim/USA M05-5 (2012).

[7] Y. Yang, Y. C. Tai, S. Siegel et al., Optimization and performance evaluation of the microPET II scanner for in vivo small-animal imaging, Phys. Med. Biol. 49, 2527 (2004).

[8] C. Grignon et al., Nuclear medical imaging using $\beta^{+} \gamma$ coincidence from ${ }^{44}$ Sc radio-nuclide with liquid xenon as detection medium, Nucl. Instr. Meth. A 571, 142 (2007).

[9] K. Shibuya et al., Annihilation photon acollinearity in PET: volunteer and phantom FDG studies, Phys. Med. Biol. 52, 5249 (2007).

[10] D.C. Oxley et al., Quantifying the Limitations of Small Animal Positron Emission Tomography, Nucl. Instr. Meth. A 604, 343 (2009).

[11] G.D. Hutchins et al., Small animal PET imaging, ILAR Jour. 49, 54 (2008).

[12] W. Enghardt et al., Charged hadron tumour therapy monitoring by means of PET., Nucl. Instr. Meth. A 525, 284 (2004).

[13] K. Parodi et al., Patient study on in-vivo verification of beam delivery and range using PET/CT imaging after proton therapy., Int. J. Radiat. Oncol. Biol. Phys. 68, 920 (2007b).

[14] F. Sommerer et al., In-beam PET monitoring of mono-energetic ${ }^{16} \mathrm{O}$ and ${ }^{12} \mathrm{C}$ beams: experiments and FLUKA simulations for homogeneous targets, Phys. Med. Biol. 54, 3979 (2009).

[15] K. Parodi et al., Clinical CT-based calculations of dose and positron emitter distributions in proton therapy using the FLUKA Monte Carlo code, Phys. Med. Biol. 52, 3369 (2007).

[16] E. Urakabe et al., Spot scanning using radioactive ${ }^{11} \mathrm{C}$ beams for heavy-ion radiotherapy, Jpn. J. Appl. Phys. 40, 2540 (2001).

[17] M. Kanazawa et al., Application of an RI-beam for cancer therapy: in-vivo verification of the ion-beam range by means of positron imaging, Nucl. Phys. A 701, 244c (2002).

[18] T. Tomitani et al., Washout studies of ${ }^{11} \mathrm{C}$ in rabbit thigh muscle implanted by secondary beams of HIMAC, Phys. Med. Biol. 48, 875 (2003).

[19] H. Mizuno et al., Washout measurement of radioisotope implanted by radioactive beams in the rabbit, Phys. Med. Biol. 48, 2269 (2003).

[20] T. Inaniwa et al., Quantitative comparison of suitability of various beams for range monitoring with induced $\beta^{+}$activity in hadron therapy, Phys. Med. Biol. 50, 1131 (2005).

[21] M.R. Bhat, Evaluated Nuclear Structure Data File (ENSDF), Nuclear Data for Science and Technology B 103, Springer-Verlag, Berlin, Germany, (1992).

[22] J.L. Humm, A. Rosenfeld and A. Del Guerra, From PET detectors to PET scanners, Eur. Jour. Nucl. Med. Mol. Imaging 30, 1574 (2003).

[23] S. Agostinelli et al., Geant4 - a simulation toolkit, Nucl. Instr. Meth. A 506, 250 (2003).

[24] D. Habs and U. Köster, Production of medical radioisotopes with high specific activity in photonuclear reactions with high intensity and large brilliance, Appl. Phys. B 103, 501 (2011).

[25] M. Pruszynski, N. S. Loktionova, D. V. Filosofov, F. Rösch, Post-elution processing of ${ }^{44} \mathrm{Ti}^{44} \mathrm{Sc}$ generator-derived ${ }^{44}$ Sc for clinical application, Appl. Radiat. Isot. 68, 1636 (2010).

[26] F. Rösch and R.P. Baum, Generator-based PET radiopharmaceuticals for molecular imaging of tumours: on the way to THERANOSTICS, Dalton Trans. 40, 6104 (2011). 
[27] G. Kanbach et al., Development and calibration of the tracking Compton/Pair telescope MEGA, Nucl. Instr. Meth. A 545, 310 (2005).

[28] A. Zoglauer et al., MEGAlib - The Medium Energy Gamma-ray Astronomy Library, New Astron. Rev. 50, 629 (2006).

[29] L. Torrisi, C. La Mela, and G. Foti, Doppler-broadening of positron annihilation in a biological environment, Q. Jour. Nucl. Med. 41, 18 (1997).

[30] K. Iwata, R. G. Greaves, and C. M. Surko, $\gamma$-ray spectra from positron annihilation on atoms and molecules, Phys. Rev. A 55, 3586 (1997).

[31] F. Poenisch et al., Attenuation and scatter correction for in-beam positron emission tomography monitoring of tumour irradiations with heavy ions, Phys. Med. Biol. 48, 2419 (2003).

[32] J. M. Ollinger, Model-based scatter correction for fully 3D PET, Phys. Med. Biol. 41, 153 (1996).

[33] S.E. Derenzo et al., Imaging properties of a positron tomograph with 280 BGO crystals, IEEE Trans. Nucl. Sci. 28, 81 (1981).

[34] M.-H. Richard, Design study of a Compton Camera for prompt-gamma imaging during ion-beam therapy, PhD thesis, Univ. Lyon (2012)

[35] H.M. Hudson et al., Accelerated image reconstruction using ordered subsets of projection data, IEEE Trans. Med. Imag. 13, 601 (1994).

[36] E.P. Visser et al., Spatial Resolution and Sensitivity of the Inveon Small-Animal PET Scanner, Jour. Nucl. Med. 50, 139 (2009).

[37] L. Eriksson et al., An investigation of sensitivity limits in PET scanners, Nucl. Instr. Meth. A 580, 836 (2007).

[38] M. Sajjad et al., Production and dosimetry of $\left[{ }^{14}\right.$ O]water for PET activation studies, Appl. Rad. Iso. 57, 607 (2002).

[39] C.-L. Lee et al., Radiation dose estimation using preclinical imaging with

${ }^{124}$ I-metaiodobenzylguanidine (MBIG) PET, Med. Phys. 37 , 4861 (2010).

[40] S. Senthamizhchelvan et al., Radiation Dosimetry of ${ }^{82} \mathrm{Rb}$ in Humans under Pharmacologic Stress, J. Nucl. Med. 52, 485 (2011).

[41] C.D. Winant et al., Investigation of dynamic SPECT measurements of the arterial input function in human subjects using simulation, phantom and human studies., Phys. Med. Biol. 57, 375 (2012).

[42] A. Lövqvist et al., Kinetics of ${ }^{76} \mathrm{Br}$-labeled anti-CEA antibodies in pigs; Aspects of dosimetry and PET imaging properties, Med. Phys. 26, 249 (1999).

[43] C.-H. Min et al., Prompt gamma measurements for locating the dose falloff region in the proton therapy, Appl. Phys. Lett. 89, 183517 (2006). 